\title{
Heterogeneity of Randomized Controlled Trials of Fecal Microbiota Transplantation in Recurrent Clostridioides difficile Infection
}

\author{
Paul Feuerstadt ${ }^{1,2}$ (1) Olga C. Aroniadis ${ }^{3} \cdot$ Felicia L. Svedlund ${ }^{4} \cdot$ Mariana Garcia $^{4} \cdot$ Laura Stong $^{5} \cdot$ Mena Boules $^{5}$. \\ Sahil Khanna ${ }^{6}$
}

Received: 7 January 2021 / Accepted: 28 June 2021 / Published online: 18 July 2021

(c) The Author(s) 2021

\begin{abstract}
Introduction Clinical trials have demonstrated the efficacy of FMT for reduction in CDI recurrences (rCDI), but this treatment and its reporting in the literature has significant heterogeneity. Recent publications (e.g., Ramai et al. in Dig Dis Sci 2020. https://doi.org/10.1007/s10620-020-06185-7) present the clinical outcomes for different FMT methodologies. However, to understand, compare, and contextualize outcomes, this heterogeneity in methods and reporting must be understood. Methods We performed a literature review of randomized controlled trials (RCTs) of FMT for rCDI to evaluate heterogeneity among trials. A methodical search between January 2010 and May 2019 of Medline, Embase, and Cochrane was conducted for studies investigating FMT in adults with rCDI. RCTs were evaluated for a variety of methodological and reporting criteria. Results Eight RCTs were identified, wherein 14 different FMT preparations were considered (each with distinct protocols for processing, storage, administration, and dosing). Sample sizes were generally small, with only two studies performing FMT in more than 100 patients. Three studies used non-FMT controls (vancomycin), while the remaining compared FMT with differing routes of administration or formulations. Across the identified studies, there was no standardized manner for reporting the timing of the FMT procedure. All studies tracked adverse events; however, follow-up periods were limited. Conclusions Considerable variability exists among RCTs, with marked differences in study design, control groups, and outcome assessment. Lack of a standard-of-care control in many trials may impact reproducibility of FMT trial outcomes in patients with rCDI. Widespread use of FMT for rCDI is still investigational; therefore, these foundational studies provide opportunities to optimize future trials.
\end{abstract}

Keywords Fecal microbiota transplantation $\cdot$ Recurrent Clostridioides difficile infection $\cdot$ Heterogeneity $\cdot$ Randomized controlled trials

Paul Feuerstadt

pfeuerstadt@gastrocenter.org

PACT-Gastroenterology Center, Hamden, CT, USA

2 Yale University School of Medicine, New Haven, CT, USA

3 Division of Gastroenterology, Renaissance School of Medicine At Stony, Brook University, Stony Brook, NY, USA

4 Exponent, Inc., Menlo Park, CA, USA

5 Ferring Pharmaceuticals, Inc, Parsippany, NJ, USA

6 Division of Gastroenterology and Hepatology, Mayo Clinic, Rochester, MN, USA

\section{Introduction}

Clostridioides difficile infection (CDI) is the most common healthcare-associated infection in the USA and is frequently complicated by recurrence, with up to $35 \%$ of patients developing recurrent CDI (rCDI) following an initial episode and over 50\% developing recurrences after two or more episodes [1-4]. Current guidelines from the Infectious Diseases Society of America (IDSA) and the Society for Healthcare Epidemiology of America (SHEA) recommend FMT after "appropriate antibiotic treatments for at least two recurrences" of CDI [5]. Fecal microbiota transplantation (FMT) utilizes the application of donor stool, from an otherwise healthy person, into the gastrointestinal tract, and has been utilized as a therapy for patients with rCDI following a treatment course with a standard-of-care 
antimicrobial. Some published investigator studies of FMT have reported high success rates in reducing future recurrences [6-8].

A number of prospective single-arm and randomized controlled trials (RCTs) have examined the use of FMT to reduce future recurrences in patients with rCDI. These studies were performed by independent study groups and report differing methodologies (e.g., varying rCDI diagnostic criteria, FMT preparation, and comparator groups). The considerable heterogeneity among these studies creates a challenge for inter-study comparison, limits the reproducibility of results, limits the possibility of pooling results or conducting meta-analyses, and may account for the observed variability in FMT outcomes. Limited reporting of key methodological components within manuscripts may also be a challenge to reproducibility [9].

The first meta-analysis of FMT for rCDI by Kassam et al. in 2013 included 11 studies and reported an overall efficacy of $89 \%$, while several subsequent meta-analyses have reported variable efficacy of FMT with results ranging from 67.7 to $92 \%$ [6, 7]. Quraishi et al. reported a resolution rate-defined as improvement of symptoms or negative $C$. difficile stool culture or toxin-of $92 \%$ across all identified studies, which were highly variable and included seven prospective and 30 retrospective designs. They observed higher resolution for lower gastrointestinal delivery (e.g., colonoscopy or retention enema), while no differences were found between fresh and frozen FMT [6]. They also showed that FMT was superior to vancomycin taper. When Tariq et al. considered a more narrow group of FMT studies, limited to only those with a clinical trial design $(n=13)$, they found an overall efficacy of $76.1 \%$; however, when they separated open-label trials from RCTs, the RCTs had an efficacy of $67.7 \%$ compared with $82.7 \%$ [10].

Different resolution rates were reported by Ramai et al. 2020 in a recent systematic review and meta-analysis of 26 studies comparing the clinical outcomes of FMT delivered via colonoscopy, capsule, enema, and nasogastric tube. Their study found that the cure rate of CDI-defined as resolution of CDI symptoms - via colonoscopy was comparable to that of capsule (94.8\% vs. $92.1 \%$ ), though superior to that of delivery via enema and nasogastric tube $(87.2 \%$ and $78.1 \%$, respectively) [8].

Variations in study methodology, reproducibility of study results, and transparency in reporting is paramount, playing a significant role in our overall foundational understanding of the efficacy of FMT in patients with rCDI and impacting our ability to develop standardized sample preparation and administration protocols. The aim of this analysis was to evaluate the heterogeneity of methodologies in RCTs of FMT for rCDI, highlighting differences and similarities between inclusion/exclusion criteria, FMT formulations, administration methods, and follow-up.

\section{Methods}

A search protocol and review strategy were developed to investigate the efficacy of therapies for rCDI. Though the initial search broadly captured multiple therapies, our final focus was on RCTs investigating FMT due to the significant heterogeneity between studies.

\section{Data Sources and Study Selection}

A methodical search for publications was conducted using the Medline, Embase, and Cochrane databases. Inclusion criteria were as follows: study investigated rCDI, published in English, investigated an adult-only ( $\geq 18$ years) population, and included a sample size greater than ten patients. Microbiota-based therapies under an Investigational New Drug Application for FDA approval and licensure were excluded. No exclusions were made based on CDI resolution criteria. All RCTs were identified for further screening.

Two reviewers independently assessed the identified abstracts based on pre-determined selection criteria and identified studies meeting the selection criteria. Initial selections were verified by the second reviewer. For studies in which the reviewers did not reach the same determination for inclusion/exclusion, the articles were reviewed for further assessment. Any discrepancies for study qualification between the two reviewers were discussed and resolved together. A flow diagram describing the identification and screening of the studies is presented in Fig. 1.

Screened RCTs were further evaluated for methodological heterogeneity, including choice of controls, inclusion/ exclusion criteria, route of FMT administration, storage, dosing, and follow-up time for efficacy and safety outcomes. The safety and efficacy outcomes from each study were not the focus of this study and were therefore not evaluated.

\section{Results}

The initial search identified 1353 articles (Fig. 1). After limiting to studies investigating FMT and screening and reviewing for eligibility criteria, eight randomized controlled trials investigating FMT were identified [11-18]. Three (37.5\%) compared FMT to a standard-of-care antimicrobial (vancomycin) control group, while most (62.5\%) compared FMT treatments with different routes of administration or formulations (Tables 1,2). Upon consideration of processing, storage, administration, and dosing, we identified a total of 


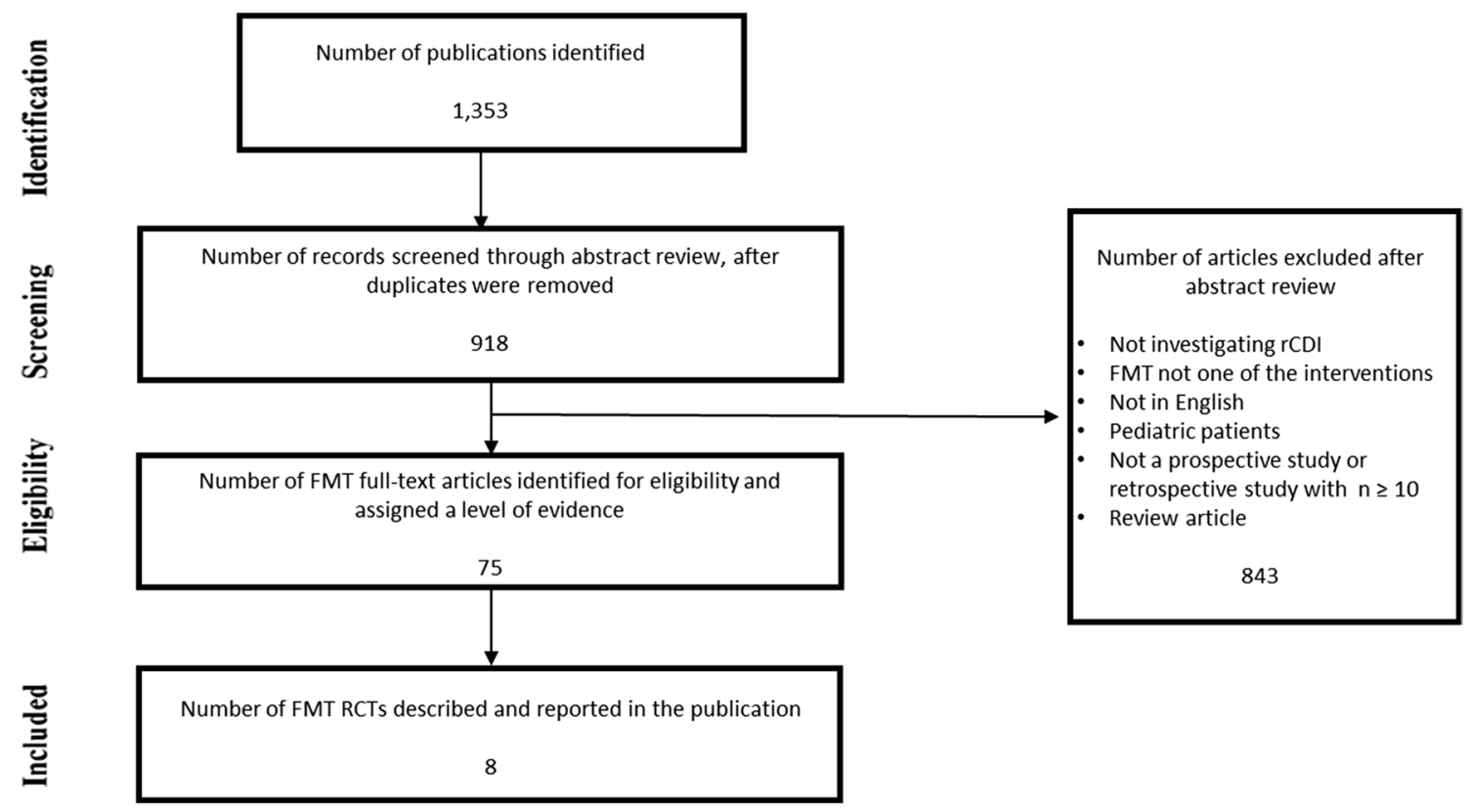

Fig. 1 Flow diagram depicting the number of records identified, included and excluded, and the reasons for exclusions

Table 1 Summary of patient populations, formulations, and study characteristics for RCTs comparing FMT to antibiotic standard-of-care (vancomycin) treatment

\begin{tabular}{|c|c|c|c|c|c|c|c|}
\hline RCT & $\begin{array}{l}\text { Patients } \\
\text { treated } \\
(\mathrm{N})\end{array}$ & $\begin{array}{l}\text { Route of adminis- } \\
\text { tration (processing) }\end{array}$ & Dosing & $\begin{array}{l}\text { Number of } \\
\text { FMT adminis- } \\
\text { trations }\end{array}$ & Patient population & $\begin{array}{l}\text { Efficacy follow- } \\
\text { up time (weeks) }\end{array}$ & $\begin{array}{l}\text { Safety } \\
\text { follow-up time } \\
\text { (weeks) }\end{array}$ \\
\hline Cammarota 2015 & 20 & $\begin{array}{l}\text { Colonoscopy } \\
\text { (fresh) }\end{array}$ & $500 \mathrm{~mL}$ & 1 or more ${ }^{a}$ & $\geq 1 \mathrm{rCDI}$ & 10.0 & 10.0 \\
\hline Hota 2016 & 16 & Enema (fresh) & $\begin{array}{l}500 \mathrm{~mL}(50 \mathrm{~g} \\
\text { stool })\end{array}$ & 1 & $\geq 1 \mathrm{rCDI}^{\mathrm{b}}$ & 17.1 & 17.1 \\
\hline van Nood 2013 & 16 & $\begin{array}{l}\text { Nasoduodenal tube } \\
\text { (fresh) }\end{array}$ & $500 \mathrm{~mL}$ & Up to 2 & $\geq 1 \mathrm{rCDI}$ & 10.0 & 10.0 \\
\hline
\end{tabular}

a Patients in whom recurrent CDI developed after the first fecal infusion were given a second fecal infusion within one week. After enrollment of the first two FMT patients, this part of the study protocol was amended, and thereafter, all subsequent patients with pseudomembranous colitis underwent repeated infusions every 3 days until the resolution of colitis

${ }^{\mathrm{b}}$ Prior to acute rCDI for randomization

14 different FMT preparations studied in the eight RCTs. In terms of number of studies, the most common FMT material was fresh stool ( $75 \%$ or $6 / 8$ studies used fresh stool), and the most common route of administration was colonoscopy ( $75 \%$ or $6 / 8$ studies used colonoscopy). When examining the number of patients treated across all eight studies, frozen stool was the most common FMT material. A total of 271 patients were treated with thawed, previously frozen stool, 210 were treated with fresh stool, and 54 were treated with lyophilized stool. Taking the same approach for FMT administration routes, we found that enema was the most common (269 patients), followed by colonoscopy (166 patients), oral pills (84 patients), and nasoduodenal tube (16 patients). Of the eight RCTs reviewed, two studies did not specify whether the procedure location was outpatient or inpatient, four studies performed FMT at outpatient clinics, and two studies performed FMT as a mix of outpatient and inpatient procedures.

None of the RCTs utilized FMT material obtained from external commercial stool banks. All studies used fecal material provided by donors identified by the patient, typically related to or cohabitating with the patient, or healthy volunteer donors. The specifics of donor screening varied among the identified RCTs. However, the majority of the studies used a combination of screening questionnaires and blood and stool testing for transmissible infections, parasites, or pathogens. Patient sample sizes across the studies were generally small, with four studies (50\%) administering 
Table 2 Summary of patient populations, formulations, and study characteristics for RCTs comparing FMT treatments with different routes of administration and formulations

\begin{tabular}{|c|c|c|c|c|c|c|c|}
\hline $\mathrm{RCT}$ & $\begin{array}{l}\text { Patients } \\
\text { treated } \\
(\mathrm{N})\end{array}$ & $\begin{array}{l}\text { Route of administra- } \\
\text { tion (processing) }\end{array}$ & Dosing & $\begin{array}{l}\text { Number of } \\
\text { FMT adminis- } \\
\text { trations }\end{array}$ & Patient population & $\begin{array}{l}\text { Efficacy follow- } \\
\text { up time (weeks) }\end{array}$ & $\begin{array}{l}\text { Safety } \\
\text { follow-up time } \\
\text { (weeks) }\end{array}$ \\
\hline \multirow[t]{3}{*}{ Jiang 2017} & 25 & Colonoscopy (fresh) & \multirow[t]{3}{*}{$50 \mathrm{~g}$ stool } & \multirow[t]{3}{*}{1} & \multirow[t]{3}{*}{$\geq 1 \mathrm{rCDI}^{\mathrm{a}}$} & \multirow[t]{3}{*}{8.6} & \multirow[t]{3}{*}{21.7} \\
\hline & 24 & Colonoscopy (frozen) & & & & & \\
\hline & 23 & $\begin{array}{l}\text { Colonoscopy (lyophi- } \\
\text { lized) }\end{array}$ & & & & & \\
\hline \multirow[t]{3}{*}{ Jiang 2018} & 17 & Oral (lyophilized) & $200 \mathrm{~g}$ stool & \multirow[t]{2}{*}{ Up to 2} & \multirow[t]{3}{*}{$\geq 2 \mathrm{rCDI}$} & \multirow[t]{3}{*}{8.6} & \multirow[t]{3}{*}{13.0} \\
\hline & 14 & Oral (lyophilized) & $100 \mathrm{~g}$ stool & & & & \\
\hline & 34 & Enema (frozen) & $500 \mathrm{~mL}$ (100 g stool) & 1 & & & \\
\hline \multirow[t]{2}{*}{ Kao 2017} & 53 & Oral (frozen) & 40 capsules & \multirow[t]{2}{*}{ Up to 2} & \multirow[t]{2}{*}{$\geq 2 \mathrm{rCDI}$} & \multirow[t]{2}{*}{12.0} & \multirow[t]{2}{*}{ Up to 13.0} \\
\hline & 52 & Colonoscopy (frozen) & $360 \mathrm{~mL}$ & & & & \\
\hline \multirow[t]{2}{*}{ Lee 2016} & 108 & Enema (frozen) & \multirow[t]{2}{*}{$50 \mathrm{~mL}$} & \multirow[t]{2}{*}{1 or more $^{\mathrm{b}}$} & \multirow{2}{*}{$\begin{aligned} \geq 2 & \text { rCDI or } \\
& 1 \text { rCDI if } \\
& \text { refractory }\end{aligned}$} & \multirow[t]{2}{*}{13.0} & \multirow[t]{2}{*}{13.0} \\
\hline & 111 & Enema (fresh) & & & & & \\
\hline Kelly 2016 & 22 & Colonoscopy (fresh) & $\begin{array}{l}300 \mathrm{~mL}(64 \pm 25 \mathrm{~g} \\
\text { stool })\end{array}$ & Up to 2 & $\geq 3 \mathrm{rCDI}$ & 8.0 & 26.1 \\
\hline
\end{tabular}

${ }^{\mathrm{a}}$ In last 12 months

${ }^{\mathrm{b}}$ On day 1, patients received FMT by enema. Patients who showed no improvement of CDI symptoms by day 4 received an additional FMT with the same donor and allocation as the original FMT between days 5 and 8. Patients not responding to two FMTs were offered repeat FMT or antibiotic therapy. A portion of the patients in each treatment group received more than five FMTs

${ }^{\mathrm{c}}$ Nonresponsive to antibiotics

specific FMT preparations to more than 50 patients, and only two studies (25\%) administering specific FMT preparations to a cohort of more than 100 patients. For any single FMT preparation, the sample size ranged from 14 to 111 patients.

The majority of the studies collected fresh donor feces and prepared it for fresh, frozen, lyophilized, or encapsulated FMT delivery by diluting the specimens in saline or water, mixing or blending the solution, and filtering or straining the solution. However, the volume of stool used and the dilution amount varied among the studies. In terms of the time from stool collection to use, some studies reported time from specimen collection to preparation, some reported time from specimen preparation to FMT, and some reported the overall time from collection to FMT. For the specimens that were frozen, lyophilized, or encapsulated and then stored before FMT, some study protocols used cryoprotectant and others did not. There was also variation in the reported maximum storage time (range 1-6 months) for frozen, lyophilized, or encapsulated specimens.

Four of the eight RCTs (50\%) used two or more episodes of rCDI as a patient enrollment criterion, whereas the remaining trials included patients with $\geq 1$ recurrence, with each of these being published in 2017 or earlier. Five of the eight RCTs $(63 \%)$ reported that patients were treated with vancomycin prior to FMT. However, the duration of vancomycin treatment varied among the studies (range 3-14 days). Six of the eight RCTs (75\%) specified a wash-out period, where antibiotic use was stopped for a specified amount of time prior to FMT. This wash-out period ranged from 24 to $72 \mathrm{~h}$ across studies.

Most studies $(6 / 8,75 \%)$ used more than one diagnostic method to confirm recurrences. The most common modality upon study enrollment was the enzyme immunoassay (EIA), which was used either independently or in combination with another diagnostic method in seven of the eight studies (87.5\%). In two studies-Jiang, 2017 and Jiang, 2018-EIA was the sole diagnostic method, but a positive result was not required for participant inclusion. Three RCTs used either EIA or polymerase chain reaction (PCR). Two RCTs accepted EIA testing of patient stools in the distant past but not within a fixed time prior to the FMT procedure. Clinical presentation (e.g., multiple liquid or unformed stools) of rCDI was included as part of the diagnostic methodology in $75 \%$ of the trials. Jiang, 2017 and Jiang, 2018 did not include clinical presentation as a diagnostic criterion in their methodology.

Among the RCTs, there was no standardized reporting for the time elapsed between rCDI diagnoses to the first FMT. Reported factors included months since last CDI episode (range $0.5-10.5$ months), duration of antibiotic use (vancomycin or metronidazole, when specified) prior to first FMT (range 6-811 days), and duration of CDI from initial diagnosis to first FMT (range 3-36 months). The follow-up time for assessment of efficacy outcomes ranged from 8.0 to 17.1 weeks. All eight RCTs tracked adverse events. However, the follow-up period was generally 
limited in the majority of RCTs, with the longest followup duration for safety assessments being up to six months in a single study (range 10-26.1 weeks).

In terms of repeat FMT administration, two studies (25\%) limited FMT to a single administration, four studies (50\%) performed no more than two FMT administrations, and the remaining two studies $(25 \%)$ did not limit the number of FMT administrations. Initially, the protocol for Cammarota et al. called for the administration of a second FMT within 1 week in patients who developed recurrent CDI after the first fecal infusion. However, after enrollment of the first two patients, the study protocol was amended so that all subsequent patients with pseudomembranous colitis underwent repeat infusions every three days until the resolution of colitis. In Lee et al., patients who showed no improvement of CDI symptoms by day 4 received an additional FMT with the same donor and allocation as the original FMT between days 5 and 8. Patients not responding to two FMTs were then offered repeat FMT or antibiotic therapy. A portion of the patients in each treatment group in the study by Lee et al. received more than five FMT administrations. However, the outcomes were not stratified based on whether the patients had recurrent or refractory CDI.

\section{Discussion}

Among the eight RCTs, there was noteworthy variation in the study structure and methodology, control groups, diagnostic method, antibiotic treatment prior to FMT, fecal transplant technique, volume of stool used, number of FMT administrations, and time to clinical outcome assessment. Differing patient populations were studied (e.g., $\geq 1$ rCDI vs. $\geq 2$ rCDI), and the time to FMT procedure was unclear, with studies using a variety of parameters prior to FMT to describe the timing of the intervention (e.g., time on vancomycin, time from most recent diagnosis). Evaluation of efficacy and safety of FMT has been assessed in other published meta-analyses, so our study concentrated on assessing the heterogeneity of the study methodologies and not on comparing outcomes. The degree of heterogeneity between studies makes it challenging to compare endpoints and reproduce outcomes. Caution should be taken in interpreting safety and efficacy across these studies and generalizing these rates.

The limitations resulting from the heterogeneity among FMT studies in patients with rCDI have been highlighted in the literature previously. Rokkas et al. conducted a metaanalysis of six RCTs investigating FMT and warned that the results should be interpreted with caution due to the heterogeneity of the examined RCTs, which differed with respect to pre-FMT preparation, number of FMT administrations, dosing, severity/ribotype of CDI, and delivery modalities [19]. Furthermore, Rokkas et al. noted that the low- to moderate-quality data (in terms of blindness and power) of the RCTs were the main limitation. Peer reviewed commentary and letters to the editor have also highlighted the lack of standardization between FMT studies and the issues associated with data assessment and comparison of outcomes. Hota et al. stated that what the procedure referred to as "FMT" is actually a variety of interventions due to the lack of standardization of variables [20]. Lagier et al. commented on a recent meta-analysis (Tariq et al. 2019) saying, "it does not make sense to do a meta-analysis when unexplained heterogeneity makes the average effect difficult to interpret and potentially misleading" [21]. To better contextualize the results and their broad applicability and reproducibility, it is important to recognize the limitation of this heterogeneity within the available literature.

Heterogeneity in the diagnostic methodology for rCDI might impact observed outcomes. As of this writing, there are no universally accepted criteria for rCDI diagnosis; the possibility that patients enrolled in the RCTs may in fact be suffering from other (non-CDI) gastrointestinal disorders (e.g., post-infection IBS) must be considered. It is documented that diagnostic method(s) have differing sensitivity and specificity, including concern that PCR might have a high rate of false positive outcomes in $C$. difficile. This was hypothesized to influence the results of a trial reported by McGovern et al., where the use of PCR testing may have misdiagnosed patients at study entry and overestimated recurrences diagnosed during the trial, thereby resulting in failure to show significant therapeutic effect when compared against placebo [22]. Due to PCR's inability to differentiate active infection from colonization, it is known to be suboptimal in diagnosing a recurrence. The optimal approach for laboratory diagnosis of CDI continues to be a point of controversy, and the IDSA/SHEA guidelines recommend more than one approach [5]. Our study found that two of the eight RCTs (25\%) tested patients' stool using an EIA test for $C$. difficile toxin but did not require a positive test result for purposes of study enrollment. This too might lead one to question whether the patients had active CDI. We believe, that moving forward, studies should do their best to adopt one set of diagnostic criteria (e.g., the IDSA/SHEA guideline recommendations). Universal diagnostic methodology would allow study results to be more easily applicable to broader patient populations and provide further guidance for community providers to identify the correct patients with rCDI and those who might benefit most from FMT.

In the RCT performed by Kelly et al., one site had a $90 \%$ placebo response rate when the patient's own stool was used as the transplant material. It was hypothesized that the reason the placebo response rate was so high was related to the length of time between re-diagnosis of rCDI and FMT administration [14]. At the site, there were prolonged periods of time-sometimes on the order of several months—-before 
the intervention, and it is believed that the prolonged time on the standard-of-care antimicrobial (e.g., vancomycin) may have effectively treated the rCDI. Thereby, the study groups (either placebo or active FMT) may have been cured prior to the intervention. Within our study, we identified much heterogeneity in reporting the "lag time" between diagnosis of rCDI and intervention. As hypothesized from the study by Kelly et al., this time period may profoundly impact outcomes following intervention. A lack of universally accepted terminology or measurement of this concept is concerning. Some studies reported months since last CDI episode, while others reported time on antimicrobials prior to first FMT, and others provided the time from initial diagnosis to first FMT.

Additionally, there was substantial variability among the study protocols in regard to antibiotic treatment and wash-out period prior to FMT. The differences in these pretransplant regimens can significantly impact FMT outcomes. Theoretically, the longer patients are on antimicrobials, the more likely the $C$. difficile infection has been sufficiently suppressed prior to FMT, meaning that FMT will likely be more successful or not play a role as the antimicrobials already eradicated the infection [23]. The IDSA/SHEA guidelines recommend a brief "induction course" of oral vancomycin for 3-4 days prior to FMT administration to reduce the burden of vegetative $C$. difficile [5]. Differences in FMT outcomes across studies could also be attributed to the variability in study protocols regarding the number of FMT administrations, as some studies limited patients to a single FMT administration, while others allowed multiple FMT administrations.

An area of weakness of the investigator-initiated trials involves heterogeneity in many elements, including donor screening. The goal for developing FMT for wide-scale application is to develop a universalized process leading to pharmaceutically generated products. Strict standards should be applied for the comprehensive evaluation of known pathogen testing, with programs designed for evaluation of new emerging threats, including viruses and multidrug-resistant organisms. Good manufacturing processes developed by pharmaceutical companies, in cooperation with federal and local regulatory and health agencies, will allow for quality control and universalized screening methods, ensuring adequate infrastructure is in place to quickly monitor for new threats and adapt protocols universally, thereby optimizing patient safety. The oversight is currently minimal for existing FMT applications.

We believe that the most important structural therapeutic factors that should be reported include: the number of episodes of CDI prior to FMT, the time since initial diagnosis to FMT, the time from the most recent $\mathrm{rCDI}$ diagnosis to FMT, the number of FMT administrations, and antibiotic treatment protocol (including wash-out period) prior to FMT. By providing this information in a more comprehensive way, the broader medical community might be better able to apply the data to their practices, understand the likelihood of patients responding to FMT, and contextualize how well their individual patients fit with the published data.

The question of most appropriate time for efficacy and safety follow-up is controversial and has not gained widespread acceptance. The gold standard for defining recurrence is a new episode within 8 weeks (56 days). Recurrence should be defined clearly, for example, the presence of CDI diarrhea within 8 weeks and a positive stool test for $C$. difficile toxin at the time of the diarrhea. Studies should include a follow-up period of at least 6 months to determine longer term safety reporting, as well as a sustained clinical response of the procedure. The pivotal trials of fidaxomicin versus vancomycin in the treatment of initial CDI or first recurrence received significant critique for only using a four-week follow-up. This short follow-up may have resulted in an underestimation of CDI recurrence rates [24, 25]. Some study follow-up durations vary and include 8,12 , and 24 weeks. This lack of universally accepted follow-up was realized in our analysis of FMT RCTs, where we observed 8.0- to 17.1-week follow-up range for tracking recurrence rates, and safety follow-up ranging from 13.0 to 26.1 weeks. Due to the lack of standardization, comparisons of recurrence rates and safety between trials must be contextualized with the followup period. Trials with a longer follow-up would likely result in an increased rate of recurrence and more adverse events. Ideally, studies should adopt universally accepted follow-up times, allowing proper comparison of outcomes.

\section{Conclusion}

The RCTs described in this study, albeit heterogeneous in reporting, have paved the way for more robust prospective clinical trial designs. The data from RCT studies and realworld evidence suggest FMT is a viable option for clinicians to offer patients who may benefit from this treatment. However, widespread use of FMT for the reduction in recurrences in patients with rCDI remains investigational; therefore, these foundational studies provide opportunities for the optimization of future trials. Future RCTs should aim for a more consistent and systematic approach and include appropriate control groups, larger sample sizes, greater standardization of product formulations and routes of administration, and universally accepted follow-up durations to track future CDI recurrences and adverse events. 


\section{Declarations}

Conflict of interest Laura Stong and Mena Boules were employed by Ferring Pharmaceuticals, Inc. at the time that the study was conducted. Mariana Garcia and Felicia L. Svedlund are employees of Exponent, Inc. Exponent has been paid fees for their consulting services performed for Ferring Pharmaceuticals, Inc. Sahil Khanna receives research support from Rebiotix, Inc (A Ferring Company). Paul Feuerstadt is a consultant for Ferring Pharmaceuticals, is on the Speakers Bureau of Merck and Co., and is a consultant for Roche diagnostics. Olga C. Aroniadis has no conflicts to disclose.

Open Access This article is licensed under a Creative Commons Attribution-NonCommercial 4.0 International License, which permits any non-commercial use, sharing, adaptation, distribution and reproduction in any medium or format, as long as you give appropriate credit to the original author(s) and the source, provide a link to the Creative Commons licence, and indicate if changes were made. The images or other third party material in this article are included in the article's Creative Commons licence, unless indicated otherwise in a credit line to the material. If material is not included in the article's Creative Commons licence and your intended use is not permitted by statutory regulation or exceeds the permitted use, you will need to obtain permission directly from the copyright holder. To view a copy of this licence, visit http://creativecommons.org/licenses/by-nc/4.0/.

\section{References}

1. Nelson W, Scott T, Boules M, Teigland C, Parente A, Unni S, Feuerstadt P. Health care resource utilization and costs of recurrent Clostridioides difficile infection in the elderly: a real-world claims analysis. J Manag Care Spec Pharm. 2021. https://doi.org/ 10.18553/jmcp.2021.20395.

2. Cornely O, Miller M, Louie T, Crook D, Gorbach S. Treatment of first recurrence of Clostridium difficile infection: fidaxomicin versus vancomycin. Clin Infect Dis 2012;55:S154-S161. https:// doi.org/10.1093/cid/cis462.

3. $\mathrm{Ng} \mathrm{K}$. Updates in the management of Clostridium difficile for adults. US Pharm 2019;44:9-12.

4. Singh T, Bedi P, Bumrah K, Singh J, Rai M, Seelam S. Updates in treatment of recurrent Clostridium difficile infection. J Clin Med Res. 2019;11:465-471. https://doi.org/10.14740/jocmr3854.

5. McDonald LC, Gerding DN, Johnson S, Bakken JS, Carroll KC, Coffin SE et al. Clinical practice guidelines for Clostridium difficile infection in adults and children: 2017 update by the Infectious Diseases Society of America (IDSA) and Society for Healthcare Epidemiology of America (SHEA). Clin Infect Dis. 2018;66:e1e48. https://doi.org/10.1093/cid/cix1085.

6. Quraishi MN, Widlak M, Bhala N, Moore D, Price M, Sharma N et al. Systematic review with meta-analysis: the efficacy of faecal microbiota transplantation for the treatment of recurrent and refractory Clostridium difficile infection. Aliment Pharmacol Ther. 2017;46:479-493. https://doi.org/10.1111/apt.14201.

7. Kassam Z, Lee CH, Yuan Y, Hunt RH. Fecal microbiota transplantation for Clostridium difficile infection: systematic review and meta-analysis. Am J Gastroenterol. 2013;108:500-508. https:// doi.org/10.1038/ajg.2013.59.

8. Ramai D, Zakhia K, Fields PJ, Ofosu A, Patel G, Shahnazarian V et al. Fecal microbiota transplantation (FMT) with colonoscopy is superior to enema and nasogastric tube while comparable to capsule for the treatment of recurrent Clostridioides difficile infection: a systematic review and meta-analysis. Dig Dis Sci. 2020. https://doi.org/10.1007/s10620-020-06185-7.
9. Bafeta A, Yavchitz A, Riveros C, Batista R, Ravaud P. Methods and reporting studies assessing fecal microbiota transplantation: a systematic review. Ann Intern Med. 2017;167:34-39. https://doi. org/10.7326/M16-2810.

10. Tariq R, Pardi DS, Bartlett MG, Khanna S. Low cure rates in controlled trials of fecal microbiota transplantation for recurrent Clostridium difficile infection: a systematic review and metaanalysis. Clin Infect Dis. 2019;68:1351-1358. https://doi.org/10. 1093/cid/ciy721.

11. van Nood E, Vrieze A, Nieuwdorp M, Fuentes S, Zoetendal EG, de Vos WM et al. Duodenal infusion of donor feces for recurrent Clostridium difficile. N Engl J Med. 2013;368:407-415. https:// doi.org/10.1056/NEJMoa1205037.

12. Cammarota G, Masucci L, Ianiro G, Bibbo S, Dinoi G, Costamagna $\mathrm{G}$ et al. Randomised clinical trial: faecal microbiota transplantation by colonoscopy vs. vancomycin for the treatment of recurrent Clostridium difficile infection. Aliment Pharmacol Ther. 2015;41:835-43. https://doi.org/10.1111/apt.13144.

13. Hota SS, Sales V, Tomlinson G, Salpeter MJ, McGeer A, Coburn $B$ et al. Oral vancomycin followed by fecal transplantation versus tapering oral vancomycin treatment for recurrent Clostridium difficile infection: an open-label, randomized controlled trial. Clin Infect Dis 2017;64:265-271. https://doi.org/10.1093/cid/ciw731.

14. Kelly CR, Khoruts A, Staley C, Sadowsky MJ, Abd M, Alani M et al. Effect of fecal microbiota transplantation on recurrence in multiply recurrent Clostridium difficile infection: a randomized trial. Ann Intern Med 2016;165:609-616. https://doi.org/10.7326/ M16-0271.

15. Lee CH, Steiner T, Petrof EO, Smieja M, Roscoe D, Nematallah A et al. Frozen vs fresh fecal microbiota transplantation and clinical resolution of diarrhea in patients with recurrent Clostridium difficile infection: a randomized clinical trial. JAMA 2016;315:142149. https://doi.org/10.1001/jama.2015.18098.

16. Jiang ZD, Ajami NJ, Petrosino JF, Jun G, Hanis CL, Shah M et al. Randomised clinical trial: faecal microbiota transplantation for recurrent Clostridum difficile infection-fresh, or frozen, or lyophilised microbiota from a small pool of healthy donors delivered by colonoscopy. Aliment Pharmacol Ther. 2017;45:899-908. https:// doi.org/10.1111/apt.13969.

17. Kao D, Roach B, Silva M, Beck P, Rioux K, Kaplan GG et al. Effect of oral capsule- vs colonoscopy-delivered fecal microbiota transplantation on recurrent Clostridium difficile infection: a randomized clinical trial. JAMA 2017;318:1985-1993. https://doi. org/10.1001/jama.2017.17077.

18. Jiang ZD, Jenq RR, Ajami NJ, Petrosino JF, Alexander AA, Ke $S$ et al. Safety and preliminary efficacy of orally administered lyophilized fecal microbiota product compared with frozen product given by enema for recurrent Clostridium difficile infection: a randomized clinical trial. PLoS One. 2018;13:e0205064. https:// doi.org/10.1371/journal.pone.0205064.

19. Rokkas T, Gisbert JP, Gasbarrini A, Hold GL, Tilg H, Malfertheiner $\mathrm{P}$ et al. A network meta-analysis of randomized controlled trials exploring the role of fecal microbiota transplantation in recurrent Clostridium difficile infection. United Eur Gastroenterol J. 2019;7:1051-1063. https://doi.org/10.1177/2050640619 854587.

20. Hota SS, Poutanen SM. Is a single fecal microbiota transplant a promising treatment for recurrent Clostridium difficile infection? Open Forum Infect Dis. 2018. https://doi.org/10.1093/ofid/ofy045.

21. Lagier JC, Million M, Raoult D. Bouillabaisse or fish soup: the limitations of meta-analysis confronted to the inconsistency of fecal microbiota transplantation studies. Clin Infect Dis. 2020;70:2454. https://doi.org/10.1093/cid/ciz707.

22. McGovern BH, Ford CB, Henn MR, Pardi DS, Khanna S, Hohmann EL et al. SER-109, an investigational microbiome drug to reduce recurrence after Clostridium difficile infection: lessons 
learned from a phase 2 trial. Clin Infect Dis. 2020. https://doi. org/10.1093/cid/ciaa387.

23. Patron RL, Hartmann CA, Allen S, Griesbach CL, Kosiorek HE, DiBaise JK et al. Vancomycin taper and risk of failure of fecal microbiota transplantation in patients with recurrent Clostridium difficile infection. Clin Infect Dis 2017;65:1214-1217.

24. Louie TJ, Miller MA, Mullane KM, Weiss K, Lentnek A, Golan $\mathrm{Y}$ et al. Fidaxomicin versus vancomycin for Clostridium difficile infection. N Engl J Med. 2011;364:422-431. https://doi.org/10. 1056/NEJMoa0910812.

25. Cornely OA, Crook DW, Esposito R, Poirier A, Somero MS, Weiss $\mathrm{K}$ et al. Fidaxomicin versus vancomycin for infection with
Clostridium difficile in Europe, Canada, and the USA: a doubleblind, non-inferiority, randomised controlled trial. Lancet Infect Dis. 2012;12:281-289. https://doi.org/10.1016/S1473-3099(11) 70374-7.

Publisher's Note Springer Nature remains neutral with regard to jurisdictional claims in published maps and institutional affiliations. 